\title{
3 Research Square \\ The Influence of Adding Traditional Chinese Medicine on COVID-19 Patients' Liver Function: A Systematic Review and Meta-analysis
}

\section{Danlei Song}

Lanzhou University First Affiliated Hospital

\section{Taobi Huang}

Lanzhou University First Affiliated Hospital

\section{Yongjian Wei}

Lanzhou University First Affiliated Hospital

\section{Yuyue Chen}

Lanzhou University First Affiliated Hospital

Yuping Wang ( $\boldsymbol{\nabla}$ wangyuping@lzu.edu.cn )

Lanzhou University First Affiliated Hospital

\section{Yongning Zhou}

Lanzhou University First Affiliated Hospital

\section{Research}

Keywords: meta-analysis, systematic review, COVID-19, liver function, Traditional Chinese Medicine

Posted Date: September 25th, 2020

DOI: https://doi.org/10.21203/rs.3.rs-77058/v1

License: (1) This work is licensed under a Creative Commons Attribution 4.0 International License. Read Full License 


\section{Abstract}

Background: Corona Virus Disease 2019 (COVID-19) is highly infectious and spreads rapidly across the world. Some patients occurred liver damage for unexplained reasons. Our study aims to explore whether Traditional Chinese Medicine (TCM) causes severe abnormal liver function compared to that of Western Medicine during the treatment process of COVID-19.

Methods: We selected eligible studies with strict inclusion and exclusion criteria after systematically searching 7 databases up to May 30, 2020. Then, the study quality was assessed by the Cochrane risk of bias tool. Finally, we conducted a meta and subgroup analysis in the random-effects model to calculate risk ratio (RR) and $95 \%$ confidence intervals $(95 \% \mathrm{Cl})$ to evaluate the risk changes after adding TCM and the influence factor to RR.

Results: A total of 2648 articles were searched, and 3 randomized controlled studies were included. Our results indicated that the occurrence of abnormal liver function had no statistical difference in the group added with TCM from Western Medicine groups. (RR=0.55[0.16,1,85], $\left.\mathrm{I}^{2}=58 \%, \mathrm{P}=0.09>0.05\right)$. We also found that the high heterogeneity existed in two subgroups of common cases and all typecases, and it was statistically significant $(I 2=76.3 \%, P=0.04<0.058$, which means the clinical type of COVID-19 may be the source of heterogeneity.

Conclusion: Our research showed that adding TCM wouldn't damage the COVID-19 patients' liver function. On the contrary, it may reduce the risk.

\section{Background}

COVID-19, with primary symptoms of cough, muscle aches, and fever, is a highly contagious viral disease damaging breathing, digestion, cardiovascular, nerves, and other systems caused by the SARS-CoV-2 virus [1,2]. In critical patients, complications such as acute respiratory distress syndrome (ARDS), shock, and septicemia may cause multiple organ dysfunction syndromes (MODS) and even death [3,4]. Up to June 2, 2020, there are 6,265,496 cases diagnosed for COVID-19 and 375,526 people have died from the disease in 188 countries and regions [5]. Though COVID-19 has slowed down in first outbreak areas such as China, Korea and Japan, the number of diagnosed cases and death are still increasing every day in the United States, Britain, India and other areas. At present, the United States has the largest number of cumulative infections and deaths. No doubt, the COVID-19 has caused severe economic and health problem worldwide. Seeking a fast and effective treatment has become an imperative global issue for the whole world.

China is the first country to report the prevalence of COVID-19 and achieved remarkable results in containing the pandemic. By looking up the latest trial diagnosis and treatment scheme publishing by National Health Commission of the People's Republic of China, we found that the treatment of COVID-19 is still symptomatic and supportive [6]. Unfortunately, there are no approved medicines and vaccines for this disease. Most of the published researches are retrospective studies and has very little guidance value in the clinic.

TCM, one of the most traditional treatment methods, is an empirical medicine applying the Taoist and Confucian philosophy to diagnose, treat, and prevent disease [7]. TCM plays a critical role in Chinese medical services. In 2018, the TCM Department provided medical services for more than 1 billion patients, accounting for $16.1 \%$ of China's total medical services [8]. Tu youyou's team won the Nobel Prize in physiology or medicine for separating artemisinin, which is the gold standard drug for malaria treatment from Artemisia annua in 2015 [9]. 
Since the 20th century, the main contribution of TCM to global medical care is providing effective chemical monomers, while there are few researches on multi-component and multi-target. It indicates that TCM still has great potential in future services [10]. Many studies show that TCM played an active role in the treatment and prevention of SARS, H1N1, and H7N9 [11,12]. Furthermore, a lot of clinical practices show that TCM is also effective to COVID-19. In China, 91\% patients of COVID-19 used TCM in treatment [13]. Studies showed that TCM had advantages in controlling progression, reducing clinical symptoms, changing nucleic acid tests and promoting physical recovery during the whole treatment process [14]. Moreover, the effect is more significant in mild patients. However, the promotion of TCM in other countries becomes difficult due to the lack of substantive evidence and effective theoretical research [15].

In clinical, medicine is a key element causes liver damage, including TCM and Western Medicine. In particular, it is difficult to identify TCM induced liver damage because of the characteristics of multi-component, multichannel, multi-target, as well as lack of pertinent laboratory indexes [16]. In this case, does TCM cause more severe liver dysfunction in COVID-19 patients compared to Western Medicine? We conducted a systematic review and meta-analysis on this issue.

\section{Materials And Methods}

\subsection{Search strategy}

This meta-analysis was based on the Preferred Reporting Items for Systematic Reviews and Meta-Analyses (PRISMA) [17]. The protocol for this study has been registered in the International Prospective Register of Systematic Reviews (PROSPERO)(Registration ID: CRD42020164915). We searched studies from 7 databases, including PubMed, EMBASE, Web of Science, Cochrane Library, CNKI (China National Knowledge Infrastructure), Wanfang and CBM (Chinese Biomedical Database) up to May 30, 2020, with no restrictions on publication type and language. In addition, we identified potential articles through references of selected researches. The above process is performed independently by two researchers. The search strategy in PubMed is shown in Supplementary Table 1.

\subsection{Inclusion and exclusion criteria}

Inclusion criteria: (1) patients: diagnosed as COVID-19; (2) samples: more than 8 patients; (2) intervention: the patients in the treatment group received TCM besides the common Western Medicine, which is similar to control group. (3) Comparison: patients in the control group received pure Western Medicine; (4) outcome measures: patients had abnormal liver function during the treatment (No matter there were laboratory examination results of liver dysfunction in the original study or not).

Exclusion criteria: (1) diagnosis and treatment experience, summary of the meeting, case report, review, and meta-analysis; (2) animal experiments; (3) incomplete data information; (4) patients with abnormal liver function that cannot be separated from those with other complications; (5) repeated publication of data.

\subsection{Data extraction and quality assessment}

The literature screening, data extraction and quality assessment were conducted independently by two reviewers. Browsing the full text is needed when the articles couldn't be included or excluded by title or abstract. 
A pre-formed data form was used to extract information, such as the first author, study type, study time, study location, number of patients, ages, sex, intervention and the dose of drugs. The primary outcome measures were the number of patients with abnormal liver function, and the secondary outcome measures were the clinical outcome of hepatic dysfunction (showed with the change of ALT, AST and total bilirubin). The quality of included studies was assessed using the Cochrane risk of bias tool. Bias risk assessment scale consists of the following seven items: random sequence generation, allocation compliance, blinding of participants and personal, blinding of outcome assessment, include outcome, data selection reporting, and other sources of bias (Figure 1). Any differences could be resolved through discussion or a third author.

\subsection{Statistical analysis}

Revman 5.1 and Stata 15.0 were used for statistical analysis. The RR and 95\% Cl were used to estimate pooled results from studies. $I^{2}$ statistics are used to assess the magnitude of heterogeneity. When $P \geq 0.05$ or $I^{2}<50 \%$ was showed, it indicated that there was no obvious heterogeneity, and the fixed-effect model should be applied for a merger. Otherwise, the random-effect model was applied. Results were considered significant statistically when the $p$-value $<0.05$. Subgroup analyses were performed to explore sources of heterogeneity. Publication bias was evaluated by Begg's and Egger's $r$ linear regression test. $\mathrm{P}<0.05$ indicating obvious publication bias.

\section{Results}

\subsection{Eligible studies}

The study selection process was showed in Figure 2. We found 2645 articles in databases, as well as 3 researches were obtained by other means. 3 RCT studies consisting of 488 patients were included in our study. The publishing time was from March 3, 2020 to May 8, 2020. The patients came from more than 20 hospitals in China. All the researches only reported the number of patients having an abnormal liver function without the liver enzymes level. The characteristic of the included studies was showed in Figure 1.

\subsection{Bias risk assessment}

The result of bias risk assessment of included studies was shown in Table 1. Ke hu et al' s study obtained high quality in each item [18]. The research of Xiaojuan Ding et al is of high quality except for some items in blinding methods [19]. Waimin Zhou et al didn't describe the method of allocation concealment and blinding [20].

\subsection{Statistical analysis}

In order to obtain relatively conservative results, we used the random effect model to conduct the meta-analysis. Figure 3 showed the combined effect size. ( $\left.R R=0.55[0.16,1,85], I^{2}=58 \%, P=0.09>0.05\right)$ and the difference was not statistically significant. This means that there was no significant difference in COVID-19 patients' abnormal liver function between the group of adding TCM and the control group. We conducted a subgroup analysis to find the source of high heterogeneity in our study.

\subsection{Subgroup analysis}

Firstly, we conducted a subgroup analysis in terms of publishing time. Ding et al's and Zhou et al' s study were published in March 2020. While the study of Ke et.al was published in May 2020. We compared the effect sides 
of two subgroups. The results of the subgroup analysis showed that there was low heterogeneity between the two subgroups, and the difference was not statistically significant $\left(I^{2}=44.8 \%, P=0.18>0.05\right)$. It showed that the incidence of the abnormal liver function of two subgroups has no significant statistical difference (Figure 4). Secondly, we classified three studies in clinical typing. Zhou et.al's study only included common cases, while the other two studies included all kinds of cases. We found that the high heterogeneity in the two subgroups was statistically significant $\left(I^{2}=76.3 \%, P=0.04<0.05\right)$. It also indicated that the clinical classification of COVID-19 might be the source of heterogeneity (Figure 5). The other two classifying factors of subgroups we pre-planned were treatment course and patients' age, however, the treatment courses of included studies were 10 14 days, and the average age of involving patients was 50 55 years old. Considering that these two subgroups have no clinical value, we finally give up conducting the subgroup analysis of them.

\subsection{Sensitivity analysis}

We conducted the sensitivity analysis by excluding anyone's study, finding that there was no influence on effect size, which indicated that the result was relatively stable and reliable.

\subsection{Publication bias}

We also assessed the publication bias. The result of Begg's Test was $P=0.296>0.05$ and the result of Egger's test was $P=0.351>0.05$. All these results meant that there was no statistically significant in publication bias (Figure 6).

\section{Discussion}

TCM played an irreplaceable role in China's treatment experience against COVID-19. It might take effect through mechanisms such as anti-inflammation, anti-virus, immune regulation, inhibition of cytokine storm and protection of target organs[21]. It is reported that the total effective rate of TCM treating COVID-19 can reach more than $90 \%$. The TCMs including Xiyanping, Reduning, Xuebijing, Shufeng Jiedu and Lianhua Qingwen have been recommended for the treatment and prevention of COVID-19 in China [6]. Published studies have shown that TCM could relieve symptoms, improve the cure rate, shorten the course, delay progression and reduce the mortality of COVID-19 patients [18,21]. TCM can also affect the laboratory indexes, resulting in the significant decrease of ESR, CRP and IL-6, and the increase of IFN- $y$. In addition, the effect of TCM is still significant in elderly and young patients [22].

The application of TCM in COVID-19 treatment mainly depends on its efficacy and safety. Most of the related studies discuss the efficacy of TCM, while the studies about adverse reactions are few. During the treatment of COVID-19, the main adverse reactions include digestive symptoms, liver and kidney dysfunction, dyslipidemia and cardiotoxicity [23]. According to the available data, $14 \%$ 53\% of COVID-19 patients have abnormal levels of ALT and AST. The incidence of liver dysfunction in severe patients is higher than mild patients [24]. A research found the changes of moderate micro vesicular steatosis, mild lobular and portal activity COVID-19 patients' liver through biopsy [25]. The key receptor for SARS-COV-2 entering the cells is ACE2. Recent studies showed that liver cells have no expression of ACE2, which suggested that abnormal liver function in COVID-19 patients is more likely caused by the drugs and cascade inflammatory response [26]. In order to solve this problem, we designed and performed this meta-analysis. As the influence of traditional Chinese medicine therapy such as 
acupuncture, physiotherapy on the liver is unclear, we only selected medicine decoction, tablets, capsules, and other TCM oral administration in this study.

After carefully screening, we finally included three RCT studies. We found that the addition of TCM did not increase or reduce the risk of abnormal liver function during COVID-19 treatment. It is consistent with the research results of Cheng Dezhong et al and Ma Zhanping et al [27,28]. We also discovered that there was no significant correlation between the addition of TCM and the risk of liver dysfunction in all type of patients. Interestingly, adding TCM could reduce the incidence of abnormal liver function in common cases. We inferred that the improvement of adding TCM on liver function might be related to the clinical classification of COVID-19. The influence of adding TCM on liver function was also different in the included studies. We conferred that different types of TCM might cause it, and it also indicated that some types of TCM might protect patients' liver function during the COVID-19 treatment process. In the research of Zhou et.al, integrated Diammonium Glycyrrhizinate Enteric-coated Capsules with Western Medicine can reduce the incidence of abnormal liver function [20]. After reviewing the literature, we found that diammonium glycyrrhizinate, the third generation extract of licorice, has a protective effect on the liver $[29,30]$. The effects of Qingfei Touxie Fuzheng recipe and Lianhua Qingwen capsule in the other two studies on liver function need to be further explored.

Of course, our research had some limitations. Firstly, we only include three studies. The limited TCM types restricted us to explore the effects of drug types on patients' abnormal liver function. Meanwhile, the influence of TCM on different patients' liver dysfunction is also unclear because of the limitation of sample capacity and individual differences. Secondly, the included studies only reported the number of patients existing abnormal liver function but not the specific changes of liver enzymes, which prevented us from examining the specific effects of TCM on the changes of liver enzymes. Regarding the above reasons, we consulted the International Registration Center for Clinical Trials up to June 2, 2020. We found that there were three studies on the therapeutic effect of TCM in COVID-19 patients, of which two cohort studies and one randomized controlled trial [31] (Supplementary Table 2). Therefore, for the problems we want to explore, it needs more high-quality studies in the future.

\section{Conclusion}

Our research shows that the addition of TCM doesn't increase the risk of abnormal liver function in COVID-19 patients. Interestingly, the beneficial effect of adding TCM on COVID-19 patients' liver function may be related to the clinical classification. Moreover, the addition of TCM is more likely to reduce the risk of abnormal liver function in common cases. Meanwhile, more high-quality researches are needed to explore this issue in the future.

\section{Abbreviations}

COVID-19: Corona Virus Disease 2019; TCM: Traditional Chinese Medicine; RR: risk ratio; Cl: confidence intervals; ARDS: acute respiratory distress syndrome; MODS: multiple organ dysfunction syndromes; PRISMA: Preferred Reporting Items for Systematic Reviews and Meta-Analyses; PROSPERO: International Prospective Register of Systematic Reviews; CNKI: China National Knowledge Infrastructure; CBM: Chinese Biomedical Database.

\section{Declarations}


Ethics approval and consent to participate: Not applicable.

Consent for publication: Not applicable.

Availability of data and material: Not applicable.

Competing interests: None.

Funding: This research received no specific grant from any funding agency, commercial or non-profit.

Authors' contributions: Study Designed, DL.S., TB. H., and YJ. W.; Methodology, TB. H.; Writing, DL. S.; Formal Analysis, YJ. W. and TB. H.; Search, YY.C., YJ. W. and TB. H.; Data extraction, YY.C. and YJ. W.; Review and editing, DL.S., TB. H., YJ. W. and YY. C.. All authors read and approved the final manuscript.

Acknowledgements: Not applicable.

\section{References}

1. Yang J, Zheng Y, Gou X, Pu K, Chen Z, Guo Q, et al. Prevalence of comorbidities and its effects in patients infected with SARS-CoV-2: a systematic review and meta-analysis. Int J Infect Dis. 2020;94: 91-95.

2. Li LQ, Huang T, Wang YQ, Wang ZP, Liang Y, Huang TB, et al. COVID-19 patients' clinical characteristics, discharge rate, and fatality rate of meta-analysis. J Med Virol. 2020 ;92(6):577-583.

3. Atzrodt CL, Maknojia I, McCarthy RDP, Oldfield TM, Po J, Ta KTL, et al. A Guide to COVID-19: a global pandemic caused by the novel coronavirus SARS-CoV-2. FEBS J. 2020.

4. Kopel J, Perisetti A, Gajendran M, Boregowda U, Goyal HA-O. Clinical Insights into the Gastrointestinal Manifestations of COVID-19. (1573-2568 (Electronic)).

5. Dong E, Du H, Gardner L. An interactive web-based dashboard to track COVID-19 in real time. (1474-4457 (Electronic)).

6. Diagnosis and Treatment Protocol for Novel Coronavirus Pneumonia (Trial Version 7). (2542-5641 (Electronic)).

7. Tang JL, Liu BY, Ma KW. Traditional Chinese medicine. Lancet. 2008;372(9654):1938-1940.

8. National Health Commission. China Health Statistical Yearbook in 2019. China Union Medical University Press; 2019 [In Chinese].

9. Tu Y. Artemisinin-A Gift from Traditional Chinese Medicine to the World (Nobel Lecture). (1521-3773 (Electronic)).

10. Wang J, Wong YK, Liao F. What has traditional Chinese medicine delivered for modern medicine? (14623994 (Electronic)).

11. Liu P, Guo Y, Qian X, Tang S, Li Z, Chen L. China's distinctive engagement in global health. (1474-547X (Electronic)).

12. Wang C, Cao B, Liu QQ, Zou ZQ, Liang ZA, Gu L, et al. Oseltamivir compared with the Chinese traditional therapy maxingshigan-yinqiaosan in the treatment of H1N1 influenza: a randomized trial. (1539-3704 (Electronic)). 
13. Li Z. Chinese medicine is on the way to fight the epidemic in the world. International Talent. 2020; 15-7. [In Chinese]

14. Ren JL, Zhang AH, Wang XJ. Traditional Chinese medicine for COVID-19 treatment. (1096-1186 (Electronic)).

15. Zhang C, Shi L, Wang F-S. Liver injury in COVID-19: management and challenges. The Lancet Gastroenterology \& Hepatology. 2020;5(5):428-30.

16. Pan X, Zhou J, Chen Y, Xie X, Rao C, Liang J, et al. Classification, hepatotoxic mechanisms, and targets of the risk ingredients in traditional Chinese medicine-induced liver injury. (1879-3169 (Electronic)).

17. [No authors listed]. The World Health Organization's decision about traditional Chinese medicine could backfire. (1476-4687 (Electronic)).

18. Pan X, Zhou J, Chen Y, Xie X, Rao C, Liang J, et al. Classification, hepatotoxic mechanisms, and targets of the risk ingredients in traditional Chinese medicine-induced liver injury. (1879-3169 (Electronic)).

19. Moher D, Liberati A, Tetzlaff J, Altman DG. Preferred reporting items for systematic reviews and metaanalyses: the PRISMA statement. (1549-1676 (Electronic)).

20. Hu K, Guan WJ, Bi Y, Zhang W, Li L, Zhang B, et al. Efficacy and Safety of Lianhuaqingwen Capsules, a repurposed Chinese Herb, in Patients with Coronavirus disease 2019: A multicenter, prospective, randomized controlled trial. (1618-095X (Electronic)).

21. Ding XJ, Zhang Y, He DC, Zhang MY,Tan YJ, Yu AR, et al. The clinical efficacy and mechanism of Qingfei Touxie Fuzheng recipe in the treatment of COVID-19. Herald of Medicine. 2020; 1-10. [In Chinese]

22. Zhou WM, Zhao FM, Li BL, Tian ZQ. Clinical value of diammonium glycyrrhizinate in the treatment of common COVID-19 patients. Chinese Journal of Virology. 2020; 36: 160-4. [In Chinese]

23. Huang YF, Bai C, He F, Xie Y, Zhou H. Review on the potential action mechanisms of Chinese medicines in treating Coronavirus Disease 2019 (COVID-19). (1096-1186 (Electronic)).

24. Zhu YL, Yang BB, Wu F. Understanding of COVID-19 in children from different angles of Chinese and Western Medicine. Chinese Traditional and Herbal Drugs. 2020; 51: 883-7. [In Chinese]

25. Huang LJ, Chen FC, Li ZH, Li P, Xiong L, Zhou WB. Safety analysis of drug treatment for COVID-19 patients. Chinese Pharmacological Bulletin. 2020; 36: 737-8. [In Chinese]

26. Zhang C, Shi L, Wang FS. Liver injury in COVID-19: management and challenges. (2468-1253 (Electronic)).

27. Xu Z, Shi L, Wang Y, Zhang J, Huang L, Zhang C, et al. Pathological findings of COVID-19 associated with acute respiratory distress syndrome. (2213-2619 (Electronic)).

28. Chai X, Hu L, Zhang Y, Han W, Lu Z, Ke A, et al. Specific ACE2 Expression in Cholangiocytes May Cause Liver Damage After 2019-nCoV Infection. bioRxiv. 2020:2020.02.03.931766.

29. Cheng DZ, Li Y. COVID-19 treated by Lianhua Qingwen Granule: a clinical analysis and a typical case report of 54 cases. World Chinese Medicine. 2020; 15: 150-4. [In Chinese]

30. Ma ZP, Lu B, Wang ZM, Yang SZ. Clinical observation of COVID-19 treated by Zhong Yan Yi Fei Jie Du Decoctio. Shaanxi Journal of Traditional Chinese Medicie. 2020; 41: 424-6. [In Chinese]

31. Li Y, Liu TY, Yan C, Xie R, Guo Z, Wang S, et al. Diammonium Glycyrrhizinate Protects against Nonalcoholic Fatty Liver Disease in Mice through Modulation of Gut Microbiota and Restoration of Intestinal Barrier. (1543-8392 (Electronic)). 
32. Gao MX, Li XL, He LL, Yang JR, Ye XH, Xiao F, et al. Diammonium Glycyrrhizinate Mitigates Liver Injury Via Inhibiting Proliferation Of NKT Cells And Promoting Proliferation Of Tregs. (1177-8881 (Electronic)).

33. [Internet] NIH. National Library of Medicine. clinicaltrials.gov. https://www.clinicaltrials.gov/

\section{Tables}

Table 1.

\begin{tabular}{|llllllll|}
\hline Study & $\begin{array}{l}\text { Random } \\
\text { sequence } \\
\text { generation }\end{array}$ & $\begin{array}{l}\text { Allocation } \\
\text { concealment }\end{array}$ & $\begin{array}{l}\text { Blinding of } \\
\text { participants } \\
\text { and } \\
\text { personal }\end{array}$ & $\begin{array}{l}\text { Blinding of } \\
\text { outcome } \\
\text { assessment }\end{array}$ & $\begin{array}{l}\text { Incomplete } \\
\text { outcome } \\
\text { data }\end{array}$ & $\begin{array}{l}\text { Selection } \\
\text { reporting }\end{array}$ & $\begin{array}{l}\text { Other } \\
\text { sources } \\
\text { of bias }\end{array}$ \\
\hline $\begin{array}{l}\text { Xiaojuan } \\
\text { Ding } \\
2020.03\end{array}$ & High & High & Unclear & Unclear & High & High & High \\
$\begin{array}{l}\text { Waimin } \\
\text { Zhou } \\
\text { 2020.03 }\end{array}$ & High & Unclear & Unclear & Unclear & Unclear & High & High \\
Ke Hu & High & High & High & High & High & High & High \\
2020.05 & & & & & & & \\
\hline
\end{tabular}

Figures 


\begin{tabular}{|c|c|c|c|c|c|c|c|}
\hline \multirow{2}{*}{$\begin{array}{c}\text { Study } \\
\text { (Authors/Month) }\end{array}$} & \multirow[t]{2}{*}{ Design } & \multirow{2}{*}{$\begin{array}{c}\text { Sample size (No.male) } \\
\text { and Severity }\end{array}$} & \multicolumn{3}{|c|}{ Intervention } & \multicolumn{2}{|c|}{ Abnormal liver function patients $(\%)$} \\
\hline & & & Characteristic & Treatment group & Control group & Treatment group & Control group \\
\hline \multirow[t]{6}{*}{ Ding (March) } & \multirow[t]{6}{*}{ RCT } & $100(78)$ & Number of subjects & 51 & 49 & $2(3.9 \%)$ & $3(6.1 \%)$ \\
\hline & & \multirow[t]{5}{*}{ (All kinds of cases) } & Age in years & $54.7 \pm 21.3$ & $50.8 \pm 23.5$ & & \\
\hline & & & $($ mean $\pm \mathrm{SD})$ & & & & \\
\hline & & & Male gender $(\%)$ & $39(76.5 \%)$ & $39(79.6 \%)$ & & \\
\hline & & & Medications & The medications of control group+Qingfei & Western medicine $\mathrm{e}^{1}$ & & \\
\hline & & & & Touxie Fuzheng Recipe (1 dose daily for $10 \mathrm{~d}$ ) & & & \\
\hline \multirow[t]{7}{*}{ Zhou(March) } & \multirow[t]{7}{*}{ RCT } & \multirow{7}{*}{$\begin{array}{l}104(60) \text { (Only } \\
\text { common cases) }\end{array}$} & Number of subjects & 52 & 52 & \multirow[t]{7}{*}{$1(1.9 \%)$} & \multirow[t]{7}{*}{$9(17.3 \%)$} \\
\hline & & & Age in years & $52.47 \pm 10.99$ & $51.11 \pm 9.87$ & & \\
\hline & & & $($ mean $\pm \mathrm{SD})$ & & & & \\
\hline & & & Male gender $(\%)$ & $32(61.5 \%)$ & $28(53.8 \%)$ & & \\
\hline & & & \multirow[t]{3}{*}{ Medications } & The medications of control group + & Western medicine ${ }^{2}$ & & \\
\hline & & & & Diammonium Glycyrrhizinate Enteric-coated & & & \\
\hline & & & & Capsules ( 3 capsules thrice daily for $14 d$ ) & & & \\
\hline \multirow[t]{7}{*}{ Ke et.al(May) } & \multirow[t]{7}{*}{ RCT } & \multirow{7}{*}{$\begin{array}{l}284(150) \text { (All kinds of } \\
\text { cases) }\end{array}$} & Number of subjects & 142 & 142 & \multirow[t]{7}{*}{$32(22.5 \%)$} & \multirow[t]{7}{*}{$32(22.5 \%)$} \\
\hline & & & Age in years & $50.4 \pm 15.2$ & $51.8 \pm 14.8$ & & \\
\hline & & & $($ mean $\pm \mathrm{SD})$ & & & & \\
\hline & & & Male gender (\%) & $79(55.6 \%)$ & $71(50.0 \%)$ & & \\
\hline & & & \multirow[t]{3}{*}{ Medications } & The medications of control group + & Western medicine ${ }^{3}$ & & \\
\hline & & & & Lianhuaqingwen (LH) capsule ( 4 capsules & & & \\
\hline & & & & thrice daily for $14 \mathrm{~d}$ ) & & & \\
\hline
\end{tabular}

Note:

1. Western medicine based on the Diagnosis and Treatment Plan for New Coronavirus Pneumonia (Trial Fifth Edition, Revision)

2. Western medicine based on the Diagnosis and Treatment Plan for New Coronavirus Pneumonia (Trial Fifth Edition)

3. Western medicine based on the Diagnosis and Treatment Protocol for Coronavirus Pneumonia (Trial version7)/ (Diagnosis and Treatment Protocol for Coronavirus Pneumonia.2020)

\section{Figure 1}

The characteristic of Included studies 


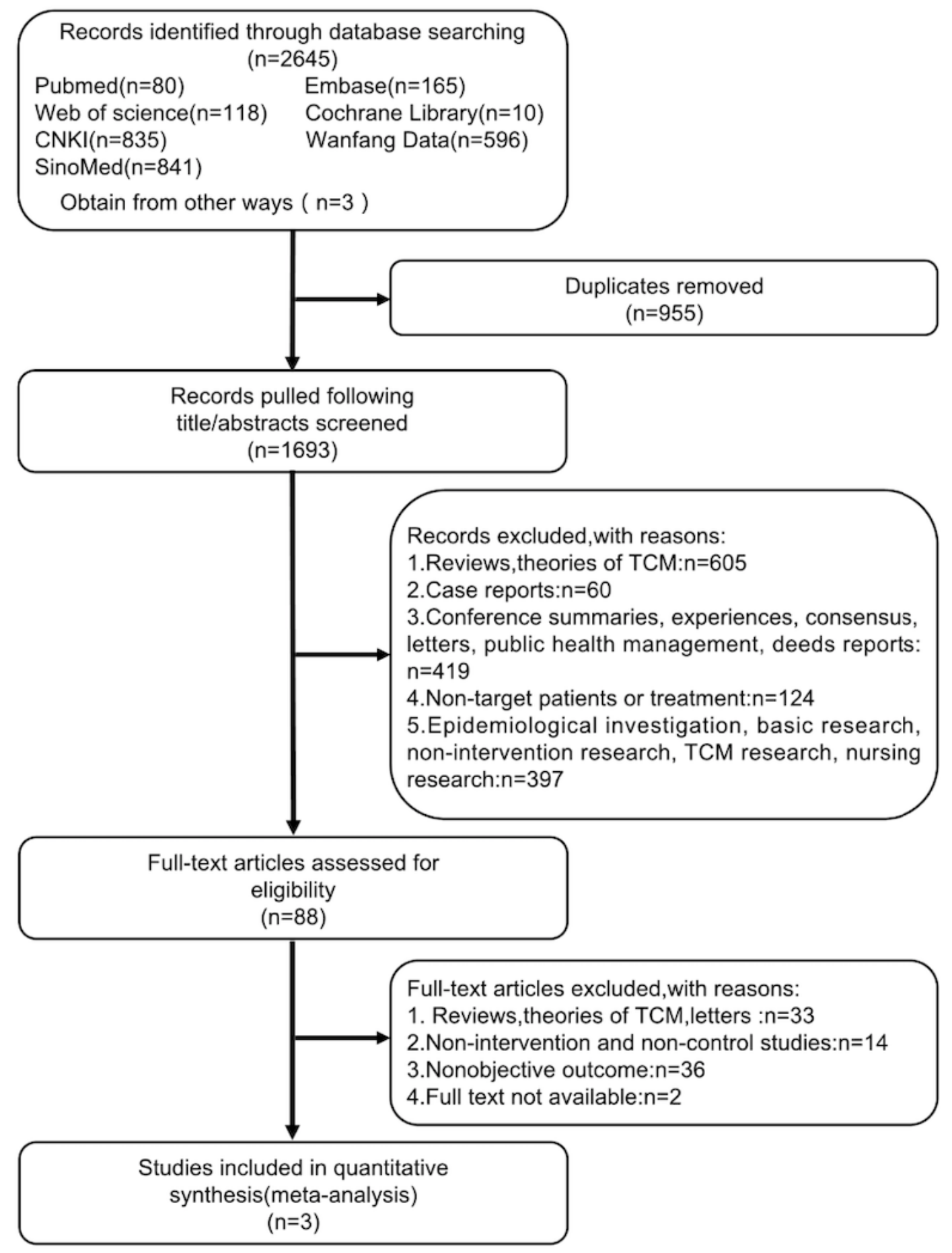

Figure 2

Flow diagram of the systematic review and meta-analysis 


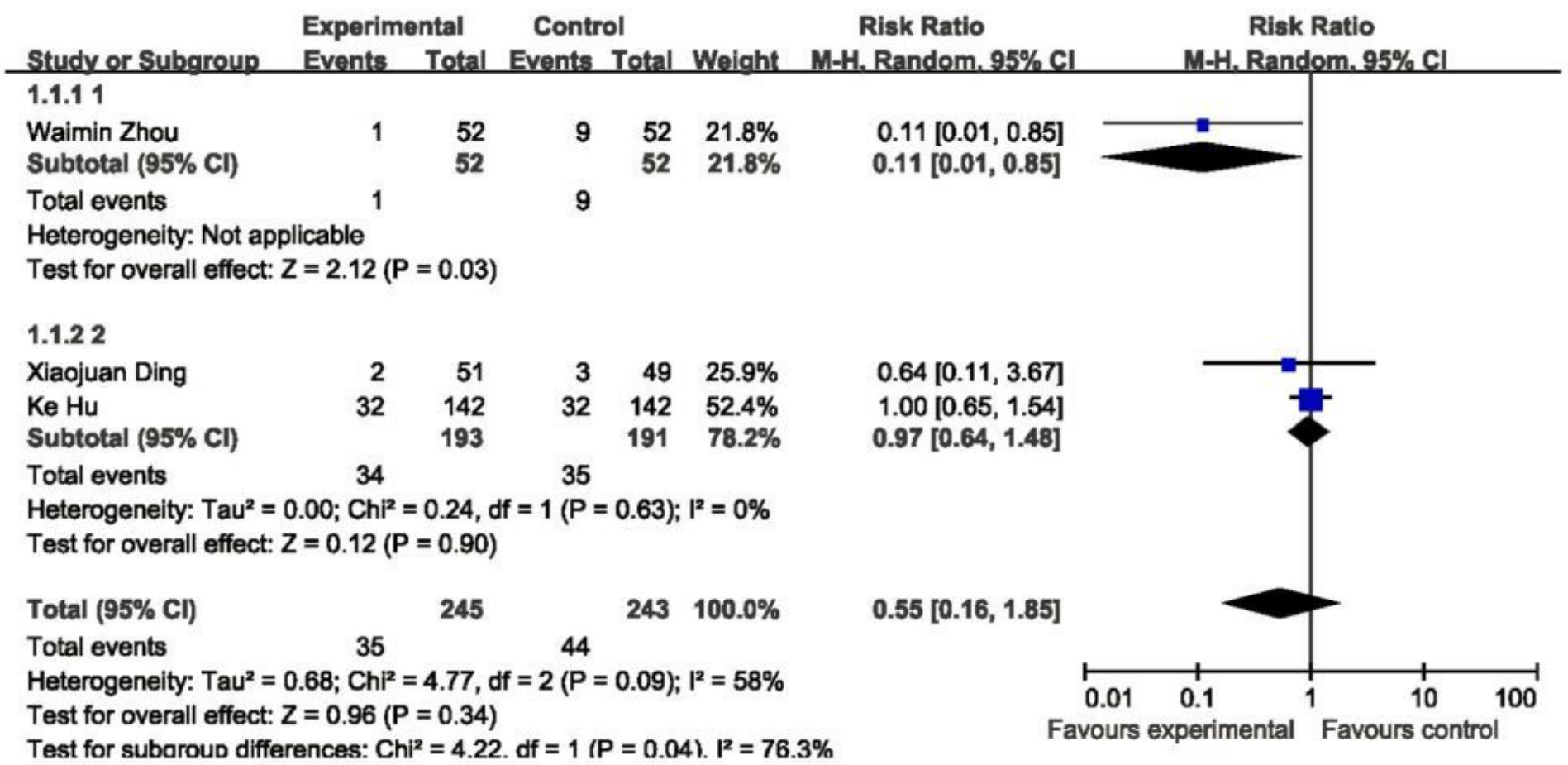

Figure 5

Subgroup analysis of different clinical classification.

Egger's publication bias plot

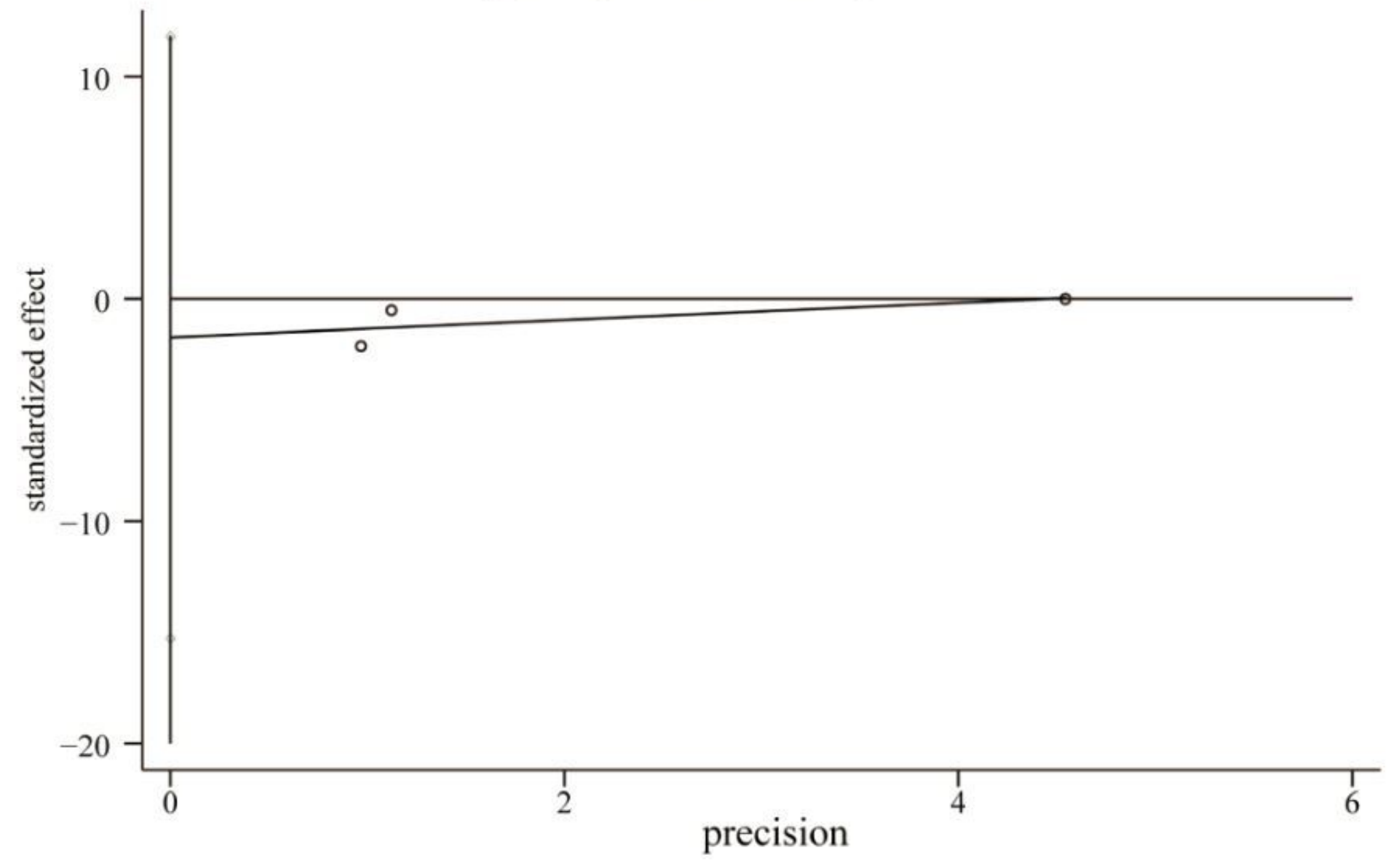


Figure 6

The evaluation of the publication bias

\section{Supplementary Files}

This is a list of supplementary files associated with this preprint. Click to download.

- supplement1.docx 